\title{
MODELING LIFE LENGTH DATA USING MIXTURE DISTRIBUTIONS
}

\author{
N. Unnikrishnan Nair*, K. G. Geetha* and P. Priya*
}

\begin{abstract}
In the present paper we characterize mixture of geometric and Waring distributions by certain identities connecting the failure rate, mean residual life and second moment of residual life. The application of the characteristic property of the geometric mixture in identification and estimation of parameters of that model is also pointed out.
\end{abstract}

Key Words and Pharase: mixture distributions, failure rate, mean residual life, geometric and Waring distributions.

\section{Introduction}

The concepts of failure rate and mean residual life are extensively used to model data on life times and to describe equipment behaviour. Let $X$ be a discrete random variable in the support of non-negative integers with probability mass function $f(x)$ and survival function

$$
R(x)=P[X \geq x] .
$$

Then the failure rate of $X$ is defined as

$$
h(x)=f(x) / R(x)
$$

and the corresponding mean residual life is

$$
r(x)=E[X-x \mid X>x]=\frac{1}{R(x+1)} \sum_{t=x+1}^{\infty} R(t) .
$$

It is well known that both $h(x)$ and $r(x)$ determine the distribution of $X$ uniquely. This fact has been exploited to characterize the distribution of $X$ based on various functional forms for $h(x)$ or $r(x)$, for example, see Nair and Hitha (1989).

In cases where simple functional forms for $h(x)$ or $r(x)$ is not available, various researchers have attempted to derive relationship between $r(x)$ and $h(x)$ that render unique representation of $f(x)$. Osaki and Li (1988) characterized the negative binomial distribution

$$
f(x)=\left(\begin{array}{c}
n-1 \\
r-1
\end{array}\right) p^{r} q^{n-1}
$$

by the identity

$$
E[X \mid X \geq x]=r p^{-1}+[(1-r)+x] p^{-1} h(x) .
$$

Received April, 1998. Revised November, 1998. Accepted February, 1999.

* Department of Statistics, Cochin University of Science and Technology, Cochin 682022, India. 
Following this, Ahmed (1991) establish that

$$
E[X \mid X \geq x]=n p+q x h(x)
$$

and

$$
E[X \mid X \geq x]=\lambda+x h(x)
$$

are respectively the characteristic properties of the binomial and Poisson distributions. More generally for the Ord family defined by

$$
\frac{f(x+1)-f(x)}{f(x)}=\frac{-(x+d)}{b_{0}+b_{1} x+b_{2} x^{2}}
$$

which includes among others, binomial, Piosson and negative binomial models, Nair and Sankaran (1991) obtained the result

$$
E[X \mid X \geq x]=\mu+\left(a_{0}+a_{1} x+a_{2} x^{2}\right) h(x)
$$

where

$$
d=\left(a_{1}-a_{2}-\mu\right) /\left(2 a_{2}+1\right) \quad \text { and } \quad b_{i}=a_{i} /\left(2 a_{2}+1\right), \quad i=0,1,2
$$

as a characterization. Further extension of these results and related references are available in Ruizz and Navarro (1994).

Nelson (1982) points out that units manufactured in different production periods may have different life distributions due to difference in design, raw materials, handling etc and it is necessary to identify production period, customer, environment etc that has poor units for remedial action on that part of the population. When the population decomposes into different such subpopulations the appropriate model of lifetime data is a mixture of distributions. Cox (1979) analyses data on failure times using mixture model by classifying the cause of failure as identified or not (see also Mendenhall and Hader (1958) and Cheng, et al. (1985)). Thus there is a case for studying mixture distributions as models in life length studies. Identification of model in such cases can be facilitated through characterization theorems involving reliability concepts. Though these characteristic properties involve reliability concepts, they can also be used for identification and inference in other data situation as well involving mixture distributions. Accordingly in the present paper we establish characterization theorems of mixture geometric and Waring distributions and point out the applications to inference of parameters. In our knowledge, no characterization of discrete mixture distributions in terms of reliability concepts have appeared in literature.

\section{Relationship between failure rate and mean residual life}

Following the notations in Section 1, we prove the following propositions.

PROPOSITION 1. X follows a mixture of geometric laws with mass function

$$
\begin{aligned}
f(x)= & \alpha p_{1} q_{1}^{x}+(1-\alpha) p_{2} q_{2}^{x}, \\
& 0<\alpha<1,0<p_{i}<1, q_{i}=1-p_{i}, \quad i=1,2, x=0,1,2, \cdots
\end{aligned}
$$


if and only if, for all $x$

$$
r(x)=\left(\frac{1}{p_{1}}+\frac{1}{p_{2}}\right)-\frac{1}{p_{1} p_{2}} h(x+1), \quad 0<p_{1}, p_{2}<1 .
$$

PROOF. For the geometric mixture (2.1) by direct calculations,

$$
h(x)=\frac{\alpha q_{1}^{x} p_{1}+(1-\alpha) q_{2}^{x} p_{2}}{\alpha q_{1}^{x}+(1-\alpha) q_{2}^{x}}
$$

and

$$
r(x)=\frac{\alpha p_{1}^{-1} q_{1}^{x+1}+(1-\alpha) p_{2}^{-1} q_{2}^{x+1}}{\alpha q_{1}^{x+1}+(1-\alpha) q_{2}^{x+1}} .
$$

From the last two equation (2.2) is verified.

Conversely, assuming (2.2), we can write it as

$$
\sum_{x+1}^{\infty} R(t)=\left(p_{1}^{-1}+p_{2}^{-1}\right) R(x+1)-p_{1}^{-1} p_{2}^{-1}[R(x+1)-R(x+2)]
$$

and hence

$$
R(x+2)-\left(2-p_{1}-p_{2}\right) R(x+1)+\left(1-p_{1}\right)\left(1-p_{2}\right) R(x)=0 .
$$

This is a homogeneous difference equation of the second order whose solution is of the form

$$
R(x)=\alpha m_{1}^{x}+\beta m_{2}^{x}
$$

where $m_{1}$ and $m_{2}$ are the roots of the auxiliary equation

$$
m^{2}-\left(2-p_{1}-p_{2}\right) m+\left(1-p_{1}\right)\left(1-p_{2}\right)=0 .
$$

Solving $m_{1}=q_{1}$ or $m_{2}=q_{2}$. Thus

$$
R(x)=\alpha q_{1}^{x}+\beta q_{2}^{x} .
$$

Since $R(0)=1, \beta=1-\alpha$ and hence the distribution is mixture geometric.

COROLLARY. When $p_{1}=p_{2}=p$, we have the relation ship

$$
r(x)=2 p^{-1}-p^{-2} h(x+1)
$$

characterizing the geometric law

$$
f(x)=q^{x} p, \quad x=0,1,2, \cdots ; 0<p<1 .
$$

PROPOSITION 2. The distribution of $X$ is a mixture of Waring distributions with

$$
\begin{aligned}
f(x)=\alpha\left(a-b_{1}\right) \frac{\left(b_{1}\right)_{x}}{(a)_{x+1}} & +(1-\alpha)\left(a-b_{2}\right) \frac{\left(b_{2}\right)_{x}}{(a)_{x+1}}, \\
x & =0,1,2, \cdots ; a>b_{1}, \quad b_{2}>0, \quad b_{1} \geq b_{2}, 0<\alpha<1
\end{aligned}
$$

where $(t)_{x}=t(t+1) \cdots(t+x-1)$ is the Pochammer's Symbol, if and only if 
$(2.4)$

$$
r(x)=\frac{\left(2 a-b_{1}-b_{2}-1\right)(a+x)}{\left(a-b_{1}-1\right)\left(a-b_{2}-1\right)}-\frac{(a+x)(a+x+1)}{\left(a-b_{1}-1\right)\left(a-b_{2}-1\right)} h(x+1)
$$

for all $x=0,1,2, \cdots$.

PROOF. Using Waring's expansion,

$$
1+\frac{a}{x+1}+\frac{a(a+1)}{(x+1)(x+2)}+\cdots=\frac{x}{x-a}
$$

it is seen that the survival function of $X$ is

$$
\begin{aligned}
& R(x)=\alpha \frac{\left(b_{1}\right)_{x}}{(a)_{x}}+(1-\alpha) \frac{\left(b_{2}\right)_{x}}{(a)_{x}} \\
& h(x)=\frac{\alpha(a+x)^{-1}\left(a-b_{1}\right)\left(b_{1}\right)_{x}+(1-\alpha)(a+x)^{-1}\left(a-b_{2}\right)\left(b_{2}\right)_{x}}{\alpha\left(b_{1}\right)_{x}+(1-\alpha)\left(b_{2}\right)_{x}}
\end{aligned}
$$

and

$$
r(x)=\frac{\alpha\left(a-b_{1}-1\right)^{-1}(a+x)\left(b_{1}\right)_{x+1}+(1-\alpha)\left(a-b_{2}-1\right)^{-1}(a+x)\left(b_{2}\right)_{x+1}}{\alpha\left(b_{1}\right)_{x+1}+(1-\alpha)\left(b_{2}\right)_{x+1}} .
$$

Thus equation (2.4) is verified.

One the other hand if we assume (2.4), proceeding as in the proof of Proposition 1, we arrive at the second order difference equation,

$$
\begin{aligned}
& (a+x)(a+x+1) R(x+2)-(a+x)\left(1+b_{1}+b_{2}+2 x\right) R(x+1) \\
& \quad+\left(b_{1}+x\right)\left(b_{2}+x\right) R(x)=0 .
\end{aligned}
$$

When (2.5) is written in the form

$$
\begin{aligned}
& \frac{(a)_{x}}{\left(b_{1}\right)_{x}}\left[\frac{\left(b_{2}\right)_{x+1}}{\left(b_{2}\right)_{x}} R(x)-\frac{(a)_{x+1}}{(a)_{x}} R(x+1)\right] \\
& \quad=\frac{(a)_{x+1}}{\left(b_{1}\right)_{x+1}}\left[\frac{\left(b_{2}\right)_{x+2}}{\left(b_{2}\right)_{x+1}} R(x+1)-\frac{(a)_{x+2}}{(a)_{x+1}} R(x+2)\right],
\end{aligned}
$$

we have the first order equation

$$
v_{x}=v_{x+1}
$$

which has the unique solution

$$
\frac{(a)_{x}}{\left(b_{1}\right)_{x}}\left[\frac{\left(b_{2}\right)_{x+1}}{\left(b_{2}\right)_{x}} R(x)-\frac{(a)_{x+1}}{(a)_{x}} R(x+1)\right]=C,
$$

where $C$ is a constant. Now setting $C=\alpha\left(b_{1}-b_{2}\right)$,

$$
\text { (2.6) } \quad R(x+1)=\frac{b_{2}+x}{a+x} R(x)+\alpha\left(b_{1}-b_{2}\right) \frac{\left(b_{1}\right)_{x}}{(a)_{x+1}}, \quad x=0,1,2, \cdots .
$$

Successive solution by iterating (2.6) for $x=0,1,2, \cdots$ leads to (2.3) and our proof is complete. 
COROLlARY. When $b_{1}=b_{2}=b$, the Waring distribution

$$
f(x)=(a-b) \frac{(b)_{x}}{(a)_{x+1}}, \quad x=0,1,2, \cdots, \quad a>b
$$

is characterized by

$$
r(x)=\frac{(2 a-2 b-1)}{(a-b-1)^{2}}(a+x)-\frac{(a+x)(a+x+1)}{(a-b-1)^{2}} h(x+1) .
$$

\section{Some Extensions}

The results in the previous section can be extended in several directions. One of them is to consider higher moments of residual life instead of the mean and explore similar relationships. For example, one may take the second factorial moment

$$
m(x)=E[(X-x)(X-x-1) \mid X>x]
$$

and characterize life distributions by its relationship with the failure rate. Such a result for the mixture of geometric laws is presented in the following proposition.

PROPOSITION 3. If $E\left(X^{2}\right)<\infty$ the identity

$$
m(x)=A h(x+1)+B
$$

where

$$
\begin{aligned}
& A=2 p_{1}^{-2} p_{2}^{-2}\left(p_{1} p_{2}-p_{1}-p_{2}\right) \\
& B=2 p_{1}^{-2} p_{2}^{-2}\left[p_{1}^{2}+p_{2}^{2}+p_{1} p_{2}\left(1-p_{1}-p_{2}\right)\right], \quad 0<p_{1}, p_{2}<1,
\end{aligned}
$$

is satisfied if and only if the distribution of $X$ is mixture geometric specified by the probability mass function

$$
f(x)=\alpha p_{1} q_{1}^{x}+(1-\alpha) p_{2} q_{2}^{x}, \quad x=0,1,2, \cdots ; 0<\alpha<1 .
$$

PROOF. First we note that

$$
\begin{aligned}
E\left[(X-x)^{2} \mid X>x\right] & =[R(x+1)]^{-1} \sum_{x+1}^{\infty}(t-x)^{2} f(x) \\
& =[R(x+1)]^{-1} \sum_{n=1}^{\infty} n^{2}(R(x+n)-R(x+n+1)) \\
& =r(x)+2[R(x+1)]^{-1} \sum_{n=1}^{\infty} n R(x+n+1) .
\end{aligned}
$$

Hence

$$
m(x)=2[R(x+1)]^{-1} \sum_{n=1}^{\infty} n R(x+n+1) .
$$

Direct calculation yield the value of $m(x)$ for the mixture geometric as 


$$
m(x)=2[R(x+1)]^{-1}\left[\alpha q_{1}^{x+2} p_{1}^{-2}+(1-\alpha) q_{2}^{x+2} p_{2}^{-2}\right],
$$

where

$$
R(x)=\alpha q_{1}^{x}+(1-\alpha) q_{2}^{x} .
$$

From (3.5) and the expression for $h(x)$, in Proposition (1), we can verify (3.1)

Conversely relationship (3.1) reduces, after some algebra, to

$$
\begin{aligned}
f(x+1)= & A(f(x)-3 f(x+1)+3 f(x+2)-f(x+3))+B(f(x) \\
& -2 f(x+1)+f(x+2))
\end{aligned}
$$

Introducing the forward shift operator $U f(x)=f(x+1)$, equation (3.5) becomes

$$
\left[A U^{3}-(3 A+B) U^{2}+(3 A+2 B+1) U-(A+B)\right] f(x)=0 .
$$

The solution of the last equation has the form

$$
f(x)=P t_{1}^{x}+Q t_{2}^{x}+R t_{3}^{x}
$$

where $t_{1}, t_{2}$ and $t_{3}$ are solutions of

$$
A t^{3}-(3 A+B) t^{2}+(3 A+2 B+1) t-(A+B)=0 .
$$

Substituting for $\mathrm{A}$ and $\mathrm{B}$ and solving,

$$
t_{1}=q_{1}, t_{2}=q_{2} \text { and } t_{3}=\left(p_{2}^{2}-p_{1}^{2}\right)\left(p_{2}^{2}-p_{1}^{2}-p_{1} p_{2}\left(p_{1}+p_{2}\right)\right)^{-1} .
$$

Thus

$$
f(x)=\alpha p_{1} q_{1}^{x}+\beta p_{2} q_{2}^{x}+R\left[\left(p_{2}^{2}-p_{1}^{2}\right) /\left(p_{2}^{2}-p_{1}^{2}-p_{1} p_{2}\left(p_{1}+p_{2}\right)\right)\right]^{x}
$$

with $\alpha=P / p_{1}$ and $\beta=Q / p_{2}$. Note that $f(x)$ cannot be a probability mass function unless $R=0$ and $\beta=1-\alpha$. This completes the proof.

Another possible extension is to increase the component densities in $f(x)$ by choosing mixing constants $\alpha_{1}, \alpha_{2}, \cdots, \alpha_{n}$ such that $\alpha_{1}+\alpha_{2}+\cdots+\alpha_{n}=1$. We present a result in the geometric case for $n=3$ in the next proposition. The proof is omitted as it is on already familiar lines.

PROPOSITION 4. The distribution of $X$ is mixture geometric laws

$$
f(x)=\sum_{1}^{3} \alpha_{i} p_{i} q_{i}^{x}, \quad x=0,1,2, \cdots
$$

where $\sum_{1}^{3} \alpha_{i}=1,0<\alpha_{i}<1$, if and only if

$$
E\left[(X-x)^{2} \mid X>x\right]=A+B h(x)+C r(x)
$$

where

$$
\begin{aligned}
& A=-2\left(p_{1} p_{2} p_{3}\right)^{-1}\left(p_{1}+p_{2}+p_{3}\right) \\
& B=2\left(p_{1} p_{2} p_{3}\right)^{-1} \\
& C=2\left(p_{1} p_{2} p_{3}\right)^{-1}\left(p_{1} p_{2}+p_{2} p_{3}+p_{3} p_{1}\right) .
\end{aligned}
$$




\section{Discussion}

Two basic problems that have to be settled while modeling life time data are (i) identification of the appropriate distribution and (ii) estimation of parameters. The results appear to be of use in settling these two issues. From Proposition 1, the graph $(h(x+1), r(x))$ is a straight line and therefore, if the estimates of $h(x+1)$ and $r(x)$ from a random sample of life lengths exhibit this property approximately, the distribution can be taken as geometric mixture. It is to be noted in this connection that, although the component geometric distributions exhibit no ageing property, their mixture has decreasing failure rate. Thus only data with decreasing failure rate that is linear in $r(x)$ can be treated as following mixture geometric law. To see the impact of the estimates of $h(x)$ and $r(x)$ in reproducing the characteristic property we have simulated random samples of various sizes for varying values of $\alpha, p_{1}$ and $p_{2}$. The estimates of $h(x)$ and $r(x)$ used are

$$
\hat{h}(x)=\frac{n_{x}-n_{x+1}}{n_{x}} \text { and } \quad \hat{r}(x)=\frac{\sum_{x+1}^{\alpha} n_{t}}{n_{x}}
$$

where $n_{x}$ is the number surviving time $x$ (number of observations corresponding to $X>x$ ) in a sample of size $n$. One such sample plot of $\widehat{h}(x+1), \widehat{r}(x)$ ) with $\alpha=0.7, p_{1}=0.85, p_{2}=0.87, n=100$ is shown in Figure 1. The sample points provide a reasonable straight line graph as expected.

In the estimation of parameters of mixture models whether classical procedures like method of moments, maximum likelihood etc. or methods specific to certain models are used, iteration is required to solve the resulting non-linear equations that give the estimates. For a discussion on the problem of estimation in geometric mixture see Harris (1983). The linear relation between $r(x)$ and $h(x+1)$ leaves scope for graphical estimation of the parameters $p_{1}$ and $p_{2}$ through the slope and intercept of the fitted line. A more

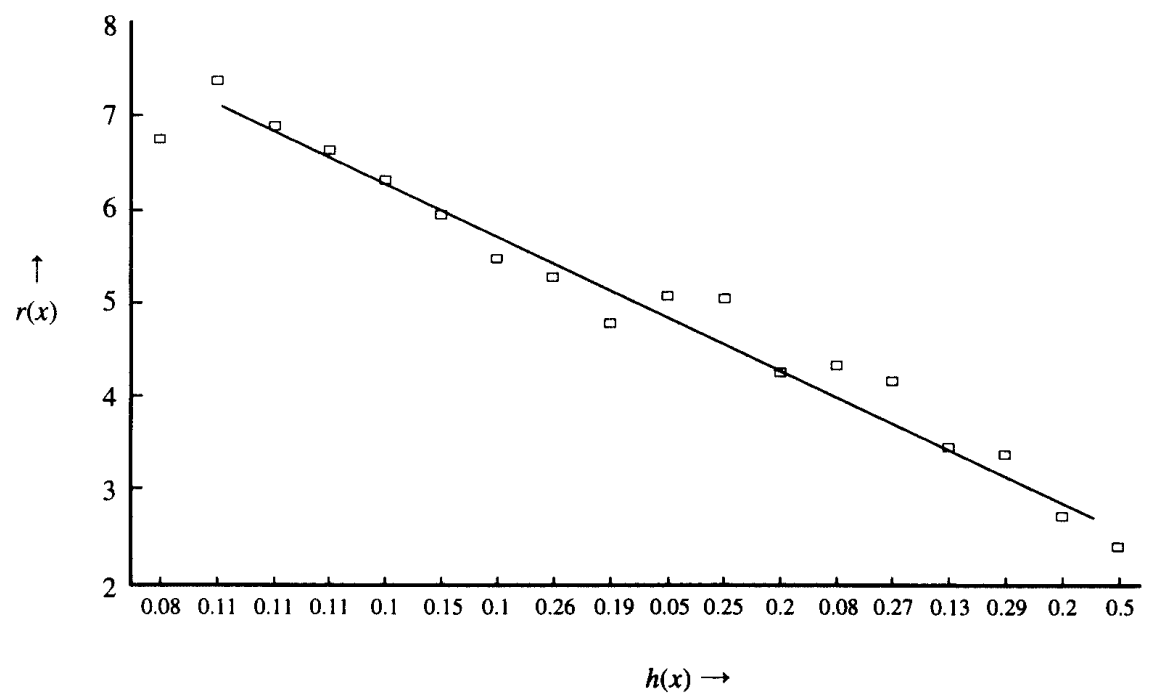

Figure 1. Relation between failure rate and mean residual life for the geometric mixture 
accurate method is the use of least squares to fit the line

$$
\widehat{r}(x)=A+B \widehat{h}(x+1)
$$

for various values of $x$ with positive frequencies and to derive estimates $p_{1}$ and $p_{2}$ from the equations

$$
\hat{A}=\hat{p}_{1}^{-1}+\hat{p}_{2}^{-1} \quad \text { and } \quad \widehat{B}=-\hat{p}_{1}^{-1} \hat{p}_{2}^{-1} .
$$

Since the mean of the geometric mixture is

$$
E(X)=\alpha q_{1} p_{1}^{-1}+(1-\alpha) q_{2} p_{2}^{-1},
$$

replacing $E(X)$ by the sample mean and then substituting for $\hat{p}_{1}$ and $\hat{p}_{2}$ we can estimate $\alpha$. In order to study the accuracy of the proposed estimates, random samples were generated from the distribution for various specified values of $p_{1}$, $p_{2}$ and $\alpha$. Some typical estimates obtained for the parameters when $p_{1}=0.35$, $p_{2}=0.85$ and $\alpha=0.7$ are shown in Table 1 .

Table 1. Estimation of the parameters of mixture geometric law

\begin{tabular}{c|c|c|c|c}
\hline Sample No. & Sample Size & $\hat{p}_{1}$ & $\hat{p}_{2}$ & $\widehat{\alpha}$ \\
\hline 1 & 100 & 0.42 & 0.82 & 0.70 \\
\hline 2 & 100 & 0.40 & 0.89 & 0.60 \\
\hline 3 & 200 & 0.31 & 0.76 & 0.60 \\
\hline 4 & 200 & 0.39 & 0.88 & 0.73 \\
\hline 5 & 400 & 0.32 & 0.83 & 0.66 \\
\hline
\end{tabular}

In moderate samples, frequencies of higher values of $X$ are absent. Such cases exhibit a large bias for the estimates. As sample size increases, the bias shows a tendency to decrease, though slowly.

Since $\hat{h}(x)$ and $\hat{r}(x)$ used in the least square procedure are ratios of random variables, the expressions for the standard errors become analytically intractable. Hence we have simulated random samples of various sizes. The mean square errors so calculated seem to be reasonable in all cases and in comparison $\widehat{\alpha}$ appears to be more unstable. For example one data on 10 simulated sample of size 200 with the above true parameter values give the following mean square error (MSE).

$$
\operatorname{MSE}\left(\hat{p}_{1}\right)=0.00133, \operatorname{MSE}\left(\hat{p}_{2}\right)=0.009089, \operatorname{MSE}(\widehat{\alpha})=0.01335 \text {. }
$$

In the case of the Waring mixture the application of the characteristic property is not straight forward as in the geometric case. The implications are being worked out.

\section{Acknowledgment}

The authors are thankful to the referee whose comments helped us a great deal to improve the paper and also to the Department of Science and Technology, Government of India, for the financial support given to this investigation. 


\section{REFERENCES}

[1] Ahmed, A. N. (1991). Characterization of beta, binomial and Poisson distributions, IEEE Transactions in Reliability, 40, 290-295.

[2] Cheng, S. W., Fu, J. C. and Sinha, S. K. (1985). An empirical procedure for estimating the parameters of mixed exponential life testing model, IEEE Transactions in Reliability, 34, 60-64.

[3] Cox, D. R. (1959). The analysis of exponentially distributed life time data with two types of failures, Journal of the Royal Statistical Society B, 21, 411-421.

[ 4 ] Harris, C. M. (1983). On finite mixtures of geometric and negative binomial distributions, Communications in Statistics A, 12, 987-1007.

[ 5 ] Mendenhall, W. and Hader, R. J. (1958). Estimation of parameters of a mixed exponential failure time distribution from censored life test data, Biometrika, 45, 504-520.

[6] Nair, N. U. and Hitha, N. (1989). Characterization of discrete models by distributions based on their partial sums, Statistics and Probability Letters, 8, 335-337.

[7] Nair, N. U. and Sankaran, P. G. (1991). Characterization of the Pearson family of distributions, IEEE Transactions in Reliability, 40, 75-77.

[8] Nelson, W. (1982). Applied life data analysis, John Wiley, New York.

[9] Osaki, S. and Li, X. (1988). Characterization of gamma and negative binomial distributions, IEEE Transactions in Reliability, 37, 379-382.

[10] Ruizz, J. M. and Navarro, J. (1994). Characterization of distribution by relationships between failure rate and mean residual life, IEEE Transactions in Reliability, 43, 640-644. 